\title{
Reflexive Rechtswissenschaft: Zur Notwendigkeit einer rechtswissenschaftlichen Didaktik
}

\begin{abstract}
Summary
The article addresses the need for a theory of legal education. It tries to demonstrate that jurisprudence needs a scientific perspective on how law is being taught. The initial point of the article is a statement of Thomas Weigend, who claims that legal education in Germany is in a state of misery. From his point of view the progress made in general didactics has failed to make a substantial impact on the system of teaching law in Germany, thus making legal education as it is still being practised at most universities an unfortunate waste of time and valuable resources. The article analyses this statement by bringing into focus how law is currently being taught in Germany. It considers the didactic approaches to jurisprudence, which includes explaining basic models and terms used in didactics, discussing the link between didactics and jurisprudence and the potential of a theory of legal education. Eventually an example is given of how to apply the theory of legal education in practice. In sum the need for a theory of legal education in order to have a scientific perspective on jurisprudence becomes obvious. At present, it is not possible to say which methods of legal education have proven most successful. Therefore, lecturers have to rely on their intuition when choosing a teaching method. So far, there are no scientific findings on the process of teaching and studying law and, consequently, on the determinants influencing a lecture. Thus, it is impossible to judge whether jurisprudence is in a state of misery and whether studying law is a wretched thing.
\end{abstract}

\section{Résumé}

Le présent article prône la nécessité d'une didactique juridique, mettant en évidence le besoin urgent d'une perspective scientifique, pour la jurisprudence, sur les méthodes d'enseignement du droit et des sciences du droit aux établissements d'enseignement supérieur. L'article part d'une critique de Thomas Weigends qui, dans les mélanges dédiés à Günther Kohlmann, décrit la « situation déplorable de l'enseignement du droit en Allemagne ». À son avis, les progrès de la didactique générale ont passé, pour l'essentiel, l'enseignement du droit en Allemagne, rendant l'enseignement universitaire du droit en Allemagne, tel qu'il est organisé pour la plupart, hélas un gaspillage de temps et de ressources précieuses. Le présent article se propose de vérifier ce diagnostic. Dans un premier temps, c'est l'organisation effective de l'enseignement aux facultés de droit en Allemagne qui sera abordée. Ensuite, ce sont les approches didactiques de l'enseignement du droit qui seront regardées de plus près. Dans ce contexte, les modèles de base et notions didactiques seront examinés, ainsi que le rapport entre didactique et sciences du droit et enfin le potentiel d'une didactique juridique, menant en conclusion à des exemplifications finales. Dans l'ensemble, l'article met en évidence la nécessité 
d'une perspective scientifique pour l'enseignement du droit. À l'heure actuelle, l'on ignore encore quelle méthode didactique per-mettra d'obtenir les meilleurs résultats auprès des étudiants. Il est p.e. impossible d'établir les déterminantes de la marge de manœuvre didactique qui sont à la base du choix d'une mé-thode d'enseignement plutôt que d'une autre. C'est pourquoi, à l'heure actuelle, le personnel enseignant doit compter sur son intuition au moment de choisir la méthode d'enseignement, faute aussi de connaissances scientifiques sur le processus d'enseignement/apprentissage pendant les études de droit et, par conséquent, sur la mise en valeur des marges de ma-nouvre didactiques pendant les cours magistraux. Tant que cela sera le cas, il est exclu de vouloir juger si l'enseignement du droit se trouve ou non dans une " situation déplorable».

An den rechtswissenschaftlichen Fakultäten im Bundesgebiet werden verschiedene Anstrengungen zur Verbesserung der Lehre unternommen. In jüngster Zeit sind Bemühungen zur Etablierung einer rechtswissenschaftlichen Didaktik zu beobachten. Ihr werden Fachtagungen, Schriftenreihen, Professuren und Forschungseinrichtungen gewidmet. Der Beitrag geht der Frage nach, ob es zur Verbesserung der Lehre im juristischen Studium tatsächlich einer Wissenschaft vom Lehren und Lernen des Rechts bedarf.

\section{Einleitung}

Thomas Weigend beschrieb vor einiger Zeit in der Festschrift für Günter Kohlmann eine „Misere der deutschen Rechtslehre“. ${ }^{1}$ Weigend konstatierte u.a., die Fortschritte der allgemeinen Didaktik seien am deutschen Rechtsunterricht im Wesentlichen vorbeigegangen, so dass der akademische Rechtsunterricht, so wie er heute noch weitgehend stattfinde, eine bedauerliche Verschwendung von Zeit und wertvollen Ressourcen darstelle. ${ }^{2}$ Diesem Befund soll nachgegangen werden. In einem ersten Zugriff ist auf die Ausbildungsrealität an juristischen Fakultäten in Deutschland zu sprechen zu kommen (II.); anschließend sind didaktische Zugänge zur Rechtslehre in den Blick zu nehmen (III.). Dabei sind didaktische Grundmodelle und Begriffe, das Verhältnis von Didaktik und Rechtswissenschaft und schließlich das Potential einer rechtswissenschaftlichen Didaktik zu erörtern. Dies soll den Boden bereiten für die abschließende Exemplifizierung (IV.).

\section{Rechtslehre und Ausbildungsrealität}

Nimmt man die Perspektive der Wissensvermittlung und Wissensaneignung ein, haben sich die Lehrveranstaltungen an den juristischen Fakultäten in der Bundesrepublik seit Jahrzehnten kaum verändert. Üblich ist Frontalunterricht. Hochschullehrer stehen Stu-

1 Thomas Weigend, Die Misere der Strafrechtslehre, in: Hans J. Hirsch/Jürgen Wolter/Uwe Brauns (Hrsg.), Festschrift für Günter Kohlmann, Köln 2003, S. 741 (741 ff.).

2 Vgl. Weigend, Misere der Strafrechtslehre (o. Fn. 1), S. 741 (754). 
dierenden gegenüber und bringen Tiefenstrukturen des Rechts näher. In den Vorlesungen zum Allgemeinen Teil des Strafgesetzbuches (StGB) werden zum Beispiel Beteiligungsformen an einer Straftat thematisiert. Den Dozenten wird dabei regelmäßig daran gelegen sein, den Studierenden Abgrenzungsprobleme zwischen den einzelnen Beteiligungsformen vor Augen zu führen. Zur Veranschaulichung werden gewöhnlich berühmte Judikate bemüht, wie etwa der ,Siriusfall“ ${ }^{3}$ in dem bekanntlich die Abgrenzung zwischen versuchtem Mord in mittelbarer Täterschaft und strafloser Anstiftung zur Selbsttötung zum Rechtsproblem wurde. Die Causa wird in dieser Untersuchung - im Rahmen der Exemplifizierung - noch einmal aufzugreifen sein. Zur visuellen Illustrierung ihrer Ausführungen setzen viele Hochschullehrer inzwischen Powerpoint-Präsentationen, gelegentlich auch noch Overheadfolien ein. In einigen Fällen erfolgt ein geleitetes Unterrichtsgespräch im Sinne der socratic method. ${ }^{4}$ Der Vorlesungsstoff wird üblicherweise häuslich vor- und nachbereitet; teilweise wird er in begleitenden Übungen und Arbeitsgemeinschaften noch einmal fallorientiert wiederholt.

Mit Blick auf den pessimistischen Befund Weigends drängt sich nun die Frage auf, ob das Festhalten an der beschriebenen Lehrtradition dazu geführt hat, dass an deutschen Universitäten schlechte Juristen ausgebildet werden. Man wird die Frage wohl verneinen müssen. Schon nach eigenem Verständnis genießen deutsche Juristen weltweit ein hohes Ansehen. Kaum ein Beitrag in der Debatte zur Reform der Juristenausbildung vergisst, auf die Wertschätzung deutscher Juristen im Ausland hinzuweisen. ${ }^{5}$ Der jüngste Bericht des Fachausschusses der Konferenz der deutschen Justizministerinnen und -minister bescheinigt ihnen im internationalen Vergleich hohe ,fachliche Kompetenz und Flexibilität“. ${ }^{6}$ Nach Peter M. Huber darf das deutsche Staatsexamen gar als „,kulturelle Errungenschaft“" gelten. ${ }^{7}$ Dies könnte dafür sprechen, dass es an der Juristenausbildung in Deutschland nichts auszusetzen gibt. Wenn nämlich ein wesentliches Produkt universitärer Lehre ist, gute Juristen auszubilden und wenn eben dieses Ziel erreicht ist,

3 BGHSt, 32, 38 ff. = BGH JZ 1984, 194 f. Näher dazu die Entscheidungsbesprechung von Ulfried Neumann, Abgrenzung von Teilnahme am Selbstmord und Tötung in mittelbarer Täterschaft, JuS 1985, 677 (677 ff.).

4 Zur Verbreitung der socratic method siehe die Beiträge in Ronald A. Brand/D. Wes Rist (Eds.), The Export of Legal Education, Farnham/Burlington 2009.

5 Vgl. Beate Merk, Impulsreferat, in: Deutscher Juristen-Fakultätentag (Hrsg.), Der „BolognaProzess" und die Juristenausbildung in Deutschland, Stuttgart 2007, S. 16 (22); Johann-Friedrich Staats, Die Ausbildung zu den Rechtsberufen in anderen Staaten Europas und der nachdenkliche Betrachter, Recht und Politik 2007, 198 (198 ff.); Heribert Hirte/Sebastian Mock, Die Juristenausbildung in Europa vor dem Hintergrund des Bologna-Prozesses, JuS 2005, Beilage 3 (14); siehe auch Heino Schöbel, Einführung des Bologna-Modells in der deutschen Juristenausbildung, in: Christian Baldus/Thomas Finkenhauer/Thomas Rüfner (Hrsg.), Juristenausbildung in Europa zwischen Tradition und Reform, S. 331 (335); kritisch dagegen Bernhard Großfeld, Die Augen der Studenten: Jurastudium zwischen Lokalisierung und Globalisierung, in: Heinz-Peter Mansel/Thomas Pfeiffer/Herbert Kronke, Festschrift für Erik Jayme, Bd. II, München 2004, S. 1103 (1106 ff.).

6 Ausschuss der Konferenz der Justizministerinnen und Justizminister zur Koordinierung der Juristenausbildung, Bericht über Möglichkeiten und Konsequenzen einer Bachelor-MasterStruktur vom 31.3.2011, http:// www.justiz.nrw.de/JM/justizpolitik/schwerpunkte/juristenausbildung/berichte/bericht2011/bericht2011.pdf (Stand: 4.12.2011), S. 149.

7 Vgl. Peter M. Huber, Beiträge zu Juristenausbildung und Hochschulrecht, Stuttgart 2010, S. 105 (107) = ZRP 2007, 188 ff. 
kann es nicht so schlecht um die Lehre des Rechts stehen. Der Bedarf an einer rechtswissenschaftlichen Didaktik drängt sich aus diesem Blickwinkel jedenfalls zunächst nicht auf.

Lenkt man jedoch den Blick vom Ausbildungsprodukt auf den Ausbildungsprozess, ergibt sich ein anderes Bild, wie einige empirische Untersuchungen veranschaulichen: Aus der Untersuchung von Stefan $\mathrm{Lueg}^{8}$ ist abzuleiten, dass die juristischen Fakultäten in Deutschland offenbar nicht allein für die Juristenausbildung verantwortlich sind. Immer noch - das hat seit den Tagen der ersten Rechtsschulen zu Bologna Tradition ${ }^{9}$ besucht ein großer Teil der Studierenden vor dem Ersten Staatsexamen ein Repetitorium. Lueg und anderen Autoren zufolge nehmen 80 bis $90 \%$ der Studierenden einen außeruniversitären Nachhilfelehrer in Anspruch. ${ }^{10}$ Im Einzelnen mögen die Motivationen für den Repetitorbesuch höchst unterschiedlich sein. ${ }^{11}$ Auch wird man nicht sagen können, dass dort die Ausbildung besser ist als an der Hochschule. ${ }^{12}$ Tatsache bleibt aber, dass bei den Studierenden offenbar der Eindruck besteht, durch den universitären Unterricht nicht genug auf das Staatsexamen vorbereitet zu sein. Aufschlüsse über mögliche Hintergründe dieser Einschätzung ergeben sich aus der Studie, die Tino Bargel, Frank Multrus und Michael Ramm im Auftrag des Bundesforschungsministeriums im Jahr 1996 an deutschen Universitäten durchgeführt haben. ${ }^{13}$ Die Untersuchung führt vor Augen, dass Studierende der Rechtswissenschaft ihren Vorlesungen deutlich geringere Lerneffekte zuschreiben als Studierende anderer Fächer. ${ }^{14}$ Die Befragten sind der Ansicht, dass sich die Lehrenden kaum über Lernfortschritte vergewissern würden. ${ }^{15}$ Noch schlechter wird die Rückmeldung auf Lernkontrollen bewertet. ${ }^{16}$ Insgesamt resümieren die Autoren, sehen sich die Studierenden während der Lehrveranstaltungen „,in die Rolle passiver Rezipienten versetzt. Ihr eigener Beitrag und ihre eigenen Anregungen scheinen bei den Lehrenden wenig gefragt. Diese Erfahrung trägt dazu bei, dass sie die Lehrveranstaltungen, insbesondere die Vorlesungen, hinsichtlich der Lernfort-

8 Stefan Lueg, Die Entstehung und Entwicklung des juristischen Privatunterrichts in den Repetitorien, Ein Beitrag zur Diskussion über die Reform der Juristenausbildung, Frankfurt am Main 1994. .

9 Siehe Thorsten Keiser, Der andere Bologna-Prozess: Ursprünge europäischer Juristenausbildung im Mittelalter, JURA 2009, 353 (356).

10 Vgl. Lueg (o. Fn. 8) Repetitorien, S. 1; Christoph Knödler, Zur heimlichen Koalition von Universität und kommerziellem Repetitor, JuS 1999, S. 1032 (1032).

11 Dazu siehe Klaus-Henning Hansen/Mario Nitsche/Manfred Walther, Der Repetitorbesuch als Strategie sozialer Anpassung, Zeitschrift für Soziologie 1975, 234 (234 ff.); Wolfgang Martin, Die Stellung des Repetitors in der deutschen Juristenausbildung, ZRP 1991, 449 (450).

12 Siehe dazu Franz Streng, Determinanten und Indikatoren von Examenserfolg und Studiendauer im Jurastudium, in: Dieter Hermann/Brigitte Tag (Hrsg.), Die universitäre Juristenausbildung, Bonn 1996, S. 32 (39); Matthias Katzenstein, Zum Status des Repetitorwesens im juristischen Studium, JURA 2006, 418 (419 ff.).

13 Tino Bargel/Frank Multrus/Michael Ramm, Das Studium der Rechtswissenschaft, Eine Fachmonographie aus studentischer Sicht, Bonn 1996.

14 Bargel/Multrus/Ramm, Studium der Rechtswissenschaft, (o. Fn.13), S. 136; vgl. auch dies., Studiensituation und studentische Orientierungen, 7. Studiensurvey an Universitäten und Fachhochschulen, Bonn 1999, S. 54.

15 Bargel/Multrus/Ramm, Studium der Rechtswissenschaft, (o. Fn. 13), S. 138.

16 Bargel/Multrus/Ramm, Studium der Rechtswissenschaft, (o. Fn. 13), S. 139. 
schritte schlechter beurteilen und diese Lehrveranstaltungen folglich seltener besuchen (...)“. ${ }^{17}$ Daraus folgern die Autoren der Studie: „Bei Überlegungen zur Verbesserung der Lehre in der Rechtswissenschaft ist darauf zu achten, wie die kommunikativen Aspekte verbessert und die Studierenden in den Lehrveranstaltungen aktiver einbezogen werden könnten“. ${ }^{18}$ Die unzureichende Einhaltung von Grundprinzipien der Lehrdidaktik und die passive Rolle der Studierenden werden als wesentliche Mängel der Lehre in der Rechtswissenschaft gesehen. ${ }^{19}$

Auch die Ergebnisse von Evaluierungen von Studium und Lehre in verschiedenen Bundesländern veranschaulichen Defizite der traditionellen Wissensvermittlung und Wissensaneignung in der juristischen Ausbildung. So wurde etwa bei der Evaluation der rechtswissenschaftlichen Lehre an Fakultäten in Süddeutschland durchgängig das Fehlen elektronischer Lehr-Lernsysteme beklagt. ${ }^{20}$ Bei der Evaluierung norddeutscher Fakultäten sprachen sich die Gutachter insbesondere für die Förderung kommunikativer Veranstaltungsformen aus, um die Anonymität des Massenstudiums einzudämmen und engere Bindungen zwischen Lehrenden und Lernenden herzustellen. ${ }^{21}$ Die These von Weigend scheint sich vor diesem Hintergrund zu erhärten.

\section{Didaktische Zugänge zur Rechtslehre}

Damit gerät die rechtswissenschaftliche Didaktik und die Frage, ob und was sie zur Verbesserung der Lehre beitragen kann, in den Blick.

\section{Didaktische Grundbegriffe und Modelle}

Um diese Frage beantworten zu können, ist eine Auseinandersetzung mit dem Wesen der Didaktik und ihrer wissenschaftssystematischen Einordnung unumgänglich. Die Verwissenschaftlichung der Didaktik lässt sich auf das programmatische Werk „Didactica Magna“ (1657) von Jan Amos Komensky („Comenius“) zurückführen. ${ }^{22}$ Comenius verstand Didaktik als „Lehrkunst, allen alles vollständig zu lehren“. Inzwischen hat sich allerdings die Ansicht durchgesetzt, dass niemand erfolgreich lehren kann, der nicht weiß, wie gelernt wird. ${ }^{23}$ Didaktik ist deshalb nach heutigem Verständnis die

17 Bargel/Multrus/Ramm, Studium der Rechtswissenschaft, (o. Fn. 13), S. 140 f.

18 Bargel/Multrus/Ramm, Studium der Rechtswissenschaft, (o. Fn. 13), S. 141.

19 Bargel/Multrus/Ramm, Studium der Rechtswissenschaft, (o. Fn. 13), S. 151.

20 Evaluationsagentur Baden-Württemberg, Evaluationsbericht Rechtswissenschaft an den Universitäten und Fachhochschulen in Baden-Württemberg 2006, http://www.evalag.de/ dedielv/ projekt01/media/pdf/evalag_berichte/jura.pdf (Stand: 4.12.2011), S. 19.

21 Vgl. Zentrale Evaluations- und Akkreditierungsagentur Hannover, Evaluation von Lehre und Studium im Fach Rechtswissenschaft an niedersächsischen Universitäten, 2000, http:// www.zeva.org/service/evadownl_pdf/Jura2.pdf(Stand: 4.12.2011), S. 24.

22 Comenius, Große Didaktik, übersetzt und herausgegeben von Andreas Flitner, Stuttgart 1998.

23 Zum „Shift from Teaching to Learning“ siehe Ulrich Welbers, The Shift from Teaching to Learning. Zur historischen Rekonstruktion eines Paradigmenwechsels, in: ders./Olaf Gaus (Hrsg.), The Shift from Teaching to Learning, Bielefeld 2005, S. 357 ff. 
Wissenschaft, Lehren auf Lernen zu beziehen. ${ }^{24}$ Sie fragt: Wer soll was von wem mit wem wo wie womit und wozu lernen ${ }^{25}$

Die Verwissenschaftlichung der Didaktik hat in der Zeit nach Comenius in Deutschland wie Europa zu einer Ausdifferenzierung der Zugänge geführt, die einer Systematisierung zugänglich sind. Die Allgemeine Didaktik untersucht in der Tradition von Comenius unabhängig von Lehrinhalten die Gesetzmäßigkeiten des Lehrens und des Lernens. Sie war zunächst eine Angelegenheit der Erziehungswissenschaft. Didaktiker wie Wolfgang Klafki, Herwig Blankertz, oder Paul Heimann sorgten in den 1950er, 1960er Jahren für eine Etablierung der Didaktik als Teildisziplin der deutschen Erziehungswissenschaft. ${ }^{26}$ Seither existiert eine kaum zu überblickende Anzahl an didaktischen Modellen. Friedrich Kron listet etwa in seinem Lehrbuch „Grundwissen Didaktik" mehr als 40 verschiedene didaktische Modelle auf. ${ }^{27}$ Als besonders einflussreich dürfen insbesondere die bildungstheoretische Didaktik, ${ }^{28}$ die lerntheoretische Didaktik $^{29}$ und die konstruktivistische Didaktik ${ }^{30}$ angesehen werden. Seit einigen Jahren bildet allerdings auch die Psychologie eigene Didaktikmodelle aus. ${ }^{31}$ Durch die Lernpsychologie wird der Erziehungswissenschaft ein Spiegel vorgehalten, wenn Lernen als Be-

24 So Johannes Wildt, Ein hochschuldidaktischer Blick auf Lehren und Lernen in gestuften Studiengängen, in Ulrich Welbers (Hrsg.), Studienreform mit Bachelor und Master, Bielefeld 2003, S. 29 (30 f.).

25 Dazu Werner Jank/Hilbert Meyer, Didaktische Modelle, 10. Auflage, Berlin 2011, S. 16 ff.; darauf bezogen Arne Pilniok/Judith Brockmann/Jan-Hendrik Dietrich, Juristische Lehre neu denken: Plädoyer für eine rechtswissenschaftliche Fachdidaktik, in: dies. (Hrsg.), Exzellente Lehre im juristischen Studium, Baden-Baden 2011, S. 9 (15).

26 Siehe ausführlich Jank/Meyer, Didaktische Modelle (o. Fn. 25), S. 29; kritisch dagegen Martin Rohland, Allgemeine Didaktik - disziplinäre Bestimmungen zwischen Willkür und Pragmatismus, Theorie und Praxis, Zeitschrift für Erziehungswissenschaft, Sonderheft 9 (2008), S. 173 (173 ff.).

27 Friedrich W. Kron, Grundwissen Didaktik, 5. Auflage, München 2008.

28 Dazu Wolfgang Klafki, Didaktische Analyse als Kern der Unterrichtsvorbereitung, Die Deutsche Schule 50 (1958), 450 (450 ff.); ders., Neue Studien zur Bildungstheorie und Didaktik: Beiträge zur kritisch-konstruktiven Didaktik, Weinheim 1985; siehe auch den Überblick bei Horst Siebert, Didaktisches Handeln in der Erwachsenenbildung, 6. Auflage, Augsburg 2009, S. $82 \mathrm{ff}$.

29 Siehe Paul Heimann/Gunter Otto/Wolfgang Schulz, Unterricht: Analyse und Planung, Hannover 1965; zu neueren Entwicklungen Jank/Meyer, Didaktische Modelle (o. Fn. 25), S. $261 \mathrm{ff}$.

30 Ausführlich dazu Kersten Reich, Konstruktivistische Didaktik, 4. Auflage, Weinheim/Basel 2008; Siebert, Didaktisches Handeln (o. Fn. 28), S. 27 ff.; Jank/Meyer, Didaktische Modelle (o. Fn. 25), S. 293 ff.; Ewald Terhart, Allgemeine Didaktik: Traditionen, Neuanfänge, Herausforderungen, Zeitschrift für Erziehungswissenschaft, Sonderheft 9 (2008), S. 14 (20 ff.).

31 Vgl. Kurt Reuser, Empirisch fundierte Didaktik - didaktisch fundierte Unterrichtsforschung, Zeitschrift für Erziehungswissenschaft, Sonderheft 9 (2008), S. 219 (220 ff.). 
dingung von Lehren verstärkt empirisch überprüft wird. ${ }^{32}$ Neben der allgemeinen Didaktik haben sich besondere Didaktiken konkretisiert. So gibt es die Schuldidaktiken, die in den 1970er Jahren entstandene Hochschuldidaktik, die speziell die universitäre Ausbildung in den Blick nimmt, sowie die sog. Bereichsdidaktiken, die sich an Erfordernissen in bestimmten Arbeits- oder Lebensbereichen orientieren. Schließlich gibt es die Fachdidaktiken. Nach früherem Verständnis wurde der Begriff der Fachdidaktik allein erziehungswissenschaftlich interpretiert, als Spezialwissenschaft vom Lehren und Lernen einzelner Schulfächer. ${ }^{33}$ In vielen Fachwissenschaften haben sich allerdings in den letzten fünf bis zehn Jahren eigenständige didaktische Modelle herausgebildet, welche Bedingungen, Logiken, Formen des fachspezifischen Lernens und Lehrens erforschen. Ein Beispiel hierfür ist die Medizindidaktik. Bundesweit sind ihr Forschungszentren und Lehrstühle gewidmet. ${ }^{34}$

Die Vielfalt der Zugänge zur Didaktik lässt erahnen: Wer die Ausbildungsrealität in der Rechtswissenschaft didaktisch verbessern will, dem muss jedenfalls klar sein, woran anzuknüpfen ist. Allein durch die Herstellung von Anschlussfähigkeit können erziehungswissenschaftliche und lernpsychologische Erkenntnisse nutzbar gemacht werden.

\section{Didaktik und Rechtswissenschaft}

Die Erforschung des Lehrens und Lernens in der Rechtswissenschaft verlangt nach alledem eine theoretische Grundierung. ${ }^{35} \mathrm{Ob}$ es hierzu der Entwicklung einer spezifisch rechtswissenschaftlichen (Fach-) Didaktik bedarf, zeigt der Blick auf das Verhältnis von Hochschuldidaktik und Rechtswissenschaft. In ihren Anfängen war die Hochschuldidaktik durchaus angetreten, auch fachspezifische Fragen der Rechtswissenschaft zu fokussieren. In Hamburg sollten beispielsweise Professuren eingerichtet werden, die ei-

32 Siehe dazu Ewald Terhart, Fremde Schwestern - Zum Verhältnis von Allgemeiner Didaktik und empirischer Lehr-Lern-Forschung, Zeitschrift für pädagogische Psychologie 16 (2002), 77 (77 ff.); Reuser, Empirisch fundierte Didaktik (o. Fn. 31), S. 219 (220); Rudolf Messner, Allgemeine Didaktik und Lehr-Lernforschung, in: Barbara Koch-Priewe/Frauke Stübig/KarlHeinz Arnold, Das Potential der Allgemeinen Didaktik, Weinheim/Basel 2007, S. 43 (43 ff.); Sigrid Blömke, Allgemeine Didaktik ohne empirische Lernforschung, in: Karl-Heinz Arnold/ Sigrid Blömke u.a., Allgemeine Didaktik und Lehr-Lernforschung, Bad Heilbrunn 2009, S. 14 ( 15 ff.).

33 So aber immer noch Jank/Meyer, Didaktische Modelle (o. Fn. 25), S. 31 ff.; Siebert verwendet im Kontext der Erwachsenenbildung den Begriff „Fachbereichsdidaktik“, vgl. ders., Didaktisches Handeln (o. Fn.28), S. 17.

34 An der medizinischen Fakultät der Eberhard Karls Universität Tübingen ist beispielsweise das Kompetenzzentrum für Medizindidaktik Baden-Württemberg eingerichtet worden. Es bietet einen „Master of Medical Education“ an, vgl. http://www.medididaktik.de (Stand: 4.12.2011); eine ähnliche Einrichtung existiert an der medizinischen Fakultät der Universität Regensburg, vgl. http://www.medizindidaktik-bayern.de. Martin Fischer hat einen Lehrstuhl für Didaktik und Ausbildungsforschung in der Medizin an der Ludwig-Maximilians-Universität München, vgl. http://www.klinikum.uni-muenchen.de/Medizinische-Klinik-Innenstadt/ Medizindidaktik/ de/index.html (Stand: 4.12.2011).

$35 \mathrm{Zu}$ dieser Forderung vgl. Judith Brockmann/Jan-Hendrik Dietrich/Arne Pilniok, Von der Lehr- zur Lernorientierung - auf dem Weg zur rechtswissenschaftlichen Fachdidaktik, JURA 2009, 579 (581); dies., Juristische Lehre neu denken (o. Fn. 25), S. 16 ff. 
nerseits an ein Hochschuldidaktisches Zentrum, andererseits an einen Fachbereich angebunden sein sollten. ${ }^{36}$ Auch in der Rechtswissenschaft gab es durchaus eine Phase hochschuldidaktischer Reflexion. So reklamierte beispielsweise Wolfgang Kilian schon 1970 eine „Didaktik des Rechts“. ${ }^{37}$ Von Michael Marx stammt die kaum bekannte Schrift „Die Didaktik des Strafrechts““. ${ }^{38}$ Letztlich blieben indes die Verständigungsund Akzeptanzprobleme zu groß und die finanziellen Ausstattungen für fachspezifische Forschungen zu gering. ${ }^{39}$ Die Hochschuldidaktik konzentrierte sich im Wesentlichen auf die Entwicklung allgemeiner Lehr-/Lern-Konzepte und -Modelle, vorwiegend aus erziehungswissenschaftlicher oder psychologischer Perspektive. In der Rechtswissenschaft stieß dies weitgehend auf Ablehnung oder vielleicht sogar auf Desinteresse. Es fand sich niemand, der sich an der Übersetzungsarbeit hochschuldidaktischer Erkenntnisse in die Rechtswissenschaft beteiligte. Jürgen Bruhn, Wolfgang Schütte und Rolf Schulmeister resümierten im März 1982 im Rückblick auf zehn Jahre hochschuldidaktischer Arbeit an der Universität Hamburg: „Bei Hochschullehrern bestand (...) vielfach die traditionell - um es vorsichtig auszudrücken - distanzierte Haltung gegenüber didaktischer Arbeit an ihrer Lehre fort." ${ }^{40}$ Als Folge dieser Entwicklung ist heute zu konstatieren: Auf der einen Seite steht die Hochschuldidaktik, die eine ganze Reihe von zu rezipierenden allgemeinen Überlegungen zum Lehren, Lernen und Prüfen in der Hochschule angestellt hat, aber - in Bezug auf die Rechtswissenschaft - kaum zu fachspezifischen Überlegungen vordringen konnte. Auf der anderen Seite wird an den juristischen Fakultäten oftmals ohne didaktische Reflexion unterrichtet. ${ }^{41}$

Ein Brückenschlag kann mit der rechtswissenschaftlichen (Fach-) Didaktik gelingen. Sie sucht die interdisziplinäre Herausforderung und kann der Hochschuldidaktik Ansprechpartner verschaffen. Sie kann die inhaltlichen Besonderheiten der Rechtswissenschaft, ihre Methoden und Rahmenbedingungen erfassen und in Bezug zu den hochschuldidaktischen Erkenntnissen setzen, um Lehr-/Lernprozesse im juristischen Studium erklärbar zu machen. Auf diese Weise bereichert sie die Rechtswissenschaft um eine wissenschaftliche Perspektive auf die Rechtslehre und ermöglicht ihr eine Selbstbeob-

36 Zur Entwicklung des Verhältnisses von Hochschuldidaktik und Rechtswissenschaft in Hamburg siehe Jürgen Bruhn/Wolfgang Schütte/Rolf Schulmeister, Hochschuldidaktik in Hamburg - 10 Jahre interdisziplinäres Zentrum für Hochschuldidaktik, uni hh reform Nr. 14 (1982).

37 Wolfgang Kilian, Zur Notwendigkeit einer Didaktik des Rechts, JuS 1970, 50 (50 ff.); ders., Ansätze einer juristischen Fachdidaktik, in: Loccumer Arbeitskreis (Hrsg.), Neue Juristenausbildung, Neuwied/Berlin 1970, S. 62 (62 ff.).

38 Michael Marx, Die Didaktik des Strafrechts, Graudruck ohne Jahresangabe, einsehbar in der Universitätsbibliothek der Ludwig-Maximilians-Universität München.

39 Dazu Jürgen Lüthje, Von der Hochschuldidaktik zur Qualitätsentwicklung, in: Marianne Merkt/Kerstin Mayrberger (Hrsg.), Die Qualität akademischer Lehre - Zur Interdependenz von Hochschuldidaktik und Hochschulentwicklung, Festschrift für Rolf Schulmeister, Band 2, Innsbruck 2007, S. 15 (19).

40 Brunn/Schütte/Schulmeister, Hochschuldidaktik in Hamburg (o. Fn. 36), S. 20.

41 Vgl. Brockmann/Dietrich/Pilniok, Rechtswissenschaftliche Fachdidaktik (o. Fn. 35), S. 579 (581). 
achtung. ${ }^{42}$ Die rechtswissenschaftliche Didaktik lässt sich insofern als reflexive Rechtswissenschaft begreifen.

\section{Potential einer rechtswissenschaftlichen Didaktik}

Die Funktionen der rechtswissenschaftlichen Didaktik sind damit benannt. Ihr Leistungspotential erschließt sich bei Betrachtung des fachspezifischen Lehr-/Lernprozesses. Als klassisches erziehungswissenschaftliches Beschreibungsraster für Lehr-/Lernprozesse gilt das sog. didaktische Dreieck. ${ }^{43}$ Lehrende, Lernende und fachlicher Inhalt sind danach die zentralen Elemente des Lehr-/Lernprozesses, der durch die jeweiligen Wechselwirkungen bestimmt wird. Aufgrund der Komplexität und Vielfalt der Faktoren, die auf die einzelnen Elemente einwirken, wird das didaktische Dreieck allgemein als konkretisierungsbedürftig angesehen. ${ }^{44}$ Bezieht man es auf Lehr-/Lernprozesse im juristischen Studium und berücksichtigt dabei die spezifischen Rahmenbedingungen der Juristenausbildung wird deutlich, dass der Lehr-/Lernprozess eine multifaktorielle Angelegenheit ist. Erst das Zusammenspiel eines fachlich, didaktisch und kommunikativ kompetenten Lehrenden, vorgebildeter, interessierter und engagierter Studierender sowie günstiger Rahmenbedingungen kann einen Lernerfolg mit hoher Wahrscheinlichkeit erzielen. Gleichzeitig liest sich dieses Modell wie eine Forschungsagenda für die rechtswissenschaftliche (Fach-) Didaktik. Mögliche Forschungsschwerpunkte wären etwa:

- „More learning, less teaching“

Nimmt man die konstruktivistische Didaktik ernst, dann steht der „Lernende“ im Mittelpunkt des Lehr-/Lernprozesses. ${ }^{45}$ Er ist gewissermaßen sein eigener Lehrer, da er so die konstruktivistische Überzeugung - sein Wissen selbstbestimmt konstruiert. Wenn man nun den berühmten ,shift from teaching to learning “46 tatsächlich vollzieht, bleibt die Frage, welche Rolle dem Lehrenden noch zukommen soll und wie er sie ausfüllen kann. Die rechtswissenschaftliche Didaktik wäre in diesem Fall z.B. dazu berufen, herauszuarbeiten, wie anregende Lernumgebungen durch die Lehrenden geschaffen werden können, um individuelle Prozesse der Wissenskonstruktion zu unterstützen.

\section{- Fachkulturforschung}

Wer über Lehrerfahrungen an einer juristischen Fakultät verfügt, dem ist bewusst, dass die Heterogenität der Studierenden gelegentlich die Gestaltung von Lehr-/Lernprozessen erschweren kann. Es bestehen unter ihnen z.B. Alters- und Erfahrungsdifferenzen

42 Dazu Arne Pilniok, Rechtswissenschaftliche Fachdidaktik als Selbstreflexion der Rechtswissenschaft, in: Jörn Griebel/Florian Gröblinghoff (Hrsg.), Von der juristischen Lehre: Erfahrungen und Denkanstöße, Baden-Baden 2011, S. 17 (17 ff.).

43 Siehe dazu Jürgen Flender, Didaktik der Hochschullehre, in: Thomas Stelzer-Rothe (Hrsg.), Kompetenzen in der Hochschullehre, 2. Auflage, Rinteln 2008, S. 170 (182 f.); Jank/Meyer, Didaktische Modelle (o. Fn. 25), S. 55.

44 Jank/Meyer, Didaktische Modelle (o. Fn. 25), S. 55.

45 Vgl. Reich, Konstruktivistische Didaktik (o. Fn. 30), S. 192 ff.

46 Siehe Wildt, Lehren und Lernen in gestuften Studiengängen (o. Fn. 24), S. 29 (37). 
oder auch nur Unterschiede in der kognitiven Leistungsfähigkeit. Die rechtswissenschaftliche Didaktik kann hier ansetzen und zur Fachkulturforschung ${ }^{47}$ werden. Sie kann Zusammenhänge zwischen Gesellschaftsstrukturen und Lernaktivitäten thematisieren. Sie kann Geschlechter-, Alters- und Differenzen der sozialen Herkunft in den Blick nehmen. Sie könnte zum Beispiel darüber Aufschluss geben, ob der Repetitorbesuch sich als Strategie sozialer Anpassung deuten lässt. ${ }^{48}$

\section{- Mediendidaktik}

In hochschuldidaktischen Beiträgen ist von ,virtuellem Lernen“ oder gar einer „virtuellen Universität" zu lesen. ${ }^{49}$ Gemeint ist der Einsatz von sog. Neuen Medien im Lehr-/ Lernprozess, schlagwortartig von „E-Learning“ oder „Blended-Learning“. ${ }^{50}$ In der deutschen Rechtslehre gehören Lernplattformen wie OLAT ${ }^{51}$ bislang noch nicht zum Standard. ${ }^{52}$ Die rechtswissenschaftliche Didaktik kann die Leistungsfähigkeit von solchen E-Learning-Angeboten und ihre Integrierbarkeit in den Lehr-/Lern-Prozess überprüfen. Denn die beste Technologie nützt wenig, wenn sie nicht Teil eines gut durchdachten didaktischen Gesamtkonzepts ist. ${ }^{53}$

\section{Didaktisch begründete Rechtslehre - eine Exemplifizierung}

Die rechtswissenschaftliche (Fach-) Didaktik darf sich nicht darauf beschränken, allein Lehr-/Lernprozesse im juristischen Studium zu erforschen und erklärbar zu machen. Ihre wesentliche Aufgabe besteht auch gerade darin, der Lehrpraxis didaktische Handlungsspielräume aufzuzeigen und ihr - ganz konkret - anwendungsorientierte Entscheidungshilfen an die Hand zu geben. In diesem Zusammenhang ist auf das eingangs erwähnte strafrechtliche Problem der Abgrenzung zwischen Anstiftung und mittelbarer Täterschaft zurückzukommen und zu exemplifizieren, wie es in die didaktische Konzeption einer Lehrveranstaltung zum Allgemeinen Teil des deutschen Strafgesetzbuches eingeordnet werden kann.

47 Zum Begriff Frank Multrus, Fachkulturen, Saarbrücken 2008; siehe daneben die Beiträge in Tino Bargel/Gerhild Framhein/Ludwig Huber/Gerhard Portele (Hrsg.), Sozialisation in der Hochschule, Beiträge für eine Auseinandersetzung zwischen Hochschuldidaktik und Sozialisationsforschung, Blickpunkt Hochschuldidaktik 37 (1975), Hamburg 1975; Bruhn/ Schütte/ Schulmeister, Hochschuldidaktik in Hamburg (o. Fn. 36), S. $53 \mathrm{f}$.

48 Vgl. hierzu Hansen/Nitsche/Walther, Der Repetitorbesuch (o. Fn. 11), S. 234 (234 ff.).

49 Siehe z.B. Rolf Schulmeister, Virtuelle Universität - virtuelles Lernen, München 2001.

50 Grundlegend dazu Rolf Schulmeister, elearning: Einsichten und Aussichten, München 2006.

51 Siehe http://www.olat.org/website/en/html/index.html (Stand: 4.12.2011).

52 So Eric Hilgendorf, Computergestützte Lehre im Recht. Entwicklungsstand und Aussichten des E-Learning in der deutschen Juristenausbildung, in: Judith Brockmann/Jan-Hendrik Dietrich/Arne Pilniok (Hrsg.), Exzellente Lehre im juristischen Studium, Baden-Baden 2011, S. 171 (171 f.); siehe auch den Ländervergleich von Christoph Revermann, eLearning in Forschung, Lehre und Weiterbildung im Ausland, TAB Hintergrundpapier Nr. 14, April 2006.

53 So zu Recht Flender, Didaktik der Hochschullehre (o. Fn. 43), S. 170 (191). 


\section{Erschließung des didaktischen Handlungsspielraums}

Die Didaktisierung einer Lehrveranstaltung durch den Lehrenden beginnt mit der Klärung seines didaktischen Handlungsspielraums. ${ }^{54}$ Wesentliche Determinanten des didaktischen Handlungsspielraums sind: die Lehrenden, die Lernenden, die Lernziele und studienrelevante Rahmenbedingungen. Ist der didaktische Handlungsspielraum erschlossen, ist der Lehrende erst in der Lage, begründete Entscheidungen für die Inhaltsauswahl und bestimmte Methoden der Stoffvermittlung zu treffen. Damit wird evident, dass es in der rechtswissenschaftlichen Didaktik nicht vordergründig darum gehen kann, wie ein Rechtsproblem den Studierenden besser zu vermitteln ist. Das würde Didaktik auf Methodik reduzieren. Die rechtswissenschaftliche Didaktik ist vielmehr aufgerufen, einen Bezugsrahmen für Methodenentscheidungen und die Inhaltsauswahl bereitzustellen.

Die Lernziele fungieren bei der Erschließung des didaktischen Entscheidungsspielraums als inhaltliche Leitplanken. Sie beschreiben den gewünschten Lernerfolg bei den Studierenden und legen fest, was, warum und wozu gelernt werden soll. ${ }^{55}$ Die Lernziele, die die Lehrenden für die einzelne Veranstaltungseinheit - in dem gewählten Beispiel die einzelne Vorlesung zum Allgemeinen Teil des StGB - formulieren, sind das Ergebnis eines komplexen Ableitungsvorgangs, der hier nur angedeutet werden kann: Auf einer Makroebene werden Lernziele vor allem durch Ausbildungs- und Prüfungsordnungen vorgegeben. Darunter fallen z.B. die Ausbildungsgesetze der Bundesländer. $\S 1$ Abs. 3 des Hamburgischen Juristenausbildungsgesetzes normiert etwa das Ausbildungsziel „gründlicher Kenntnisse der rechtlichen Regelungen, ihrer Entstehung und ihrer Systematik sowie des Gebrauchs rechtswissenschaftlicher Methoden“. Es soll damit im juristischen Studium nicht nur auf die Ansammlung von Fachwissen, sondern auch auf dogmatische Kompetenz und Methodenbewusstsein ankommen. ${ }^{56}$ Eine auf das materielle Strafrecht bezogene Konkretisierung dieser Anforderungen findet sich $\S 1$ Abs. 2 der Verordnung über Prüfungsgegenstände, nämlich dass zu den Prüfungsgegenständen des Pflichtfachs Strafrecht jedenfalls auch der zweite Abschnitt aus dem Allgemeinen Teil des StGB (,Die Tat“) gehört. Auf der Mesoebene werden die Lernziele für die Lehrveranstaltung über das Semester konkretisiert. Im Fall der Vorlesung zum StGB AT werden die Lehrenden am Ende von den Studierenden auch ein vertieftes Strukturverständnis über Beteiligungsformen an einer Straftat erwarten, ein Verständnis, das die Studierenden nicht nur in die Lage versetzt, vertretbare Falllösungen zu erarbeiten, sondern ihnen auch gleichzeitig erlaubt, einzelne Judikate oder Beiträge im Schrifttum kritisch zu hinterfragen. Um eben dieses Verständnis zu erzeugen, wird man auf der Mikroebene, also auf der hier interessierenden Ebene der einzelnen Veranstal-

54 Dazu und zum Folgenden Flender, Didaktik der Hochschullehre (o. Fn. 43), S. 170 (198 ff.); Friedemann Schulz von Thun, Wie gestalte ich meine Vorlesung und halte die Hörerschaft und mich selbst bei Laune, in: Marianne Merkt/Kerstin Mayrberger (Hrsg.), Die Qualität akademischer Lehre - Zur Interdependenz von Hochschuldidaktik und Hochschulentwicklung, Festschrift für Rolf Schulmeister, Band 2, Innsbruck 2007, S. 115 (117ff.).

55 Vgl. Jank/Meyer, Didaktische Modelle (o. Fn. 25), S. 51; Siebert, Didaktisches Handeln (o. Fn. 28), S. $136 \mathrm{ff}$.

56 Dazu zuletzt Bernd Rüthers, Wozu auch noch Methodenlehre? Die Grundlagenlücken im Jurastudium, JuS 2011, 865 (865 f.). 
tungseinheit, als Lernziel formulieren können, dass die Studierenden einzelne Beteiligungsformen voneinander abgrenzen können. Auf diese Weise gerät nun wieder das Problem der Abgrenzung von mittelbarer Täterschaft und Anstiftung in den Blick.

Hat die Lehrperson die Lernziele definiert, muss sie sich bei der Erschließung des didaktischen Handlungsspielraums noch Gedanken über die übrigen Determinanten machen. Bei der Betrachtung der Lernenden muss sie sich beispielsweise deren Vorwissen und deren Heterogenität vor Augen führen. In dem hier aufgeworfenen Beispiel haben es die Lehrenden mit einer Anfängervorlesung zu tun, bei der - schon aufgrund der hohen Teilnehmerzahl - die Heterogenität der Studierenden besonders hoch ist. ${ }^{57}$ Auch kann nur begrenzt Vorwissen aktiviert werden, möglicherweise haben die Studiereden allerdings ein Propädeutikum oder begleitende Arbeitsgemeinschaften besucht. Ferner bestimmen gewisse Rahmenbedingungen den didaktischen Handlungsspielraum. Dazu können etwa die Zahl der Teilnehmer, die Veranstaltungsform, die Dauer der Veranstaltung oder technische Kapazitäten zählen. Schließlich müssen die Lehrenden ihre eigene Rolle im Lehr-/Lernprozess für sich definieren, was ihnen insbesondere eine Analyse der eigenen fachlichen und didaktischen Kompetenzen abverlangt. In der Didaktik wird dies als subjektive Lehrkonzeption bezeichnet. ${ }^{58}$

\section{Methodenentscheidungen}

Im Anschluss an die Festlegung des didaktischen Entscheidungsspielraums können einerseits konkrete Inhalte ausgewählt und andererseits Methodenentscheidungen begründet werden. Dies betrifft etwa die Auswahl elektronischer Hilfsmittel oder die Gestaltung von Lernmaterialien. Bei der Inhaltsauswahl könnte eine Lehrperson im Fall der Vorlesung zum StGB AT z.B. dazu neigen, die Abgrenzung zwischen mittelbarer Täterschaft und Teilnahme anhand der von Fritjof Haft vorgeschlagenen ,Normalfallmethode“ zu vermitteln. ${ }^{59} \mathrm{Haft}$ wirft der Strafrechtslehre eine geradezu pathologische Lehrbuchkriminalität vor und favorisiert deshalb das „Lernen am Normalfall“. ${ }^{60}$ Vice versa könnte sich die Lehrperson dafür entscheiden, die Abgrenzungsfragen anhand eben einer dieser Entscheidungen wie dem Siriusfall zu thematisieren, weil sie z.B. der Ansicht ist, dass die Judikate die Abgrenzungsfragen besonders gut veranschaulichen und sich deshalb leicht ins Langzeitgedächtnis aufnehmen lassen.

57 Zu Bewältigungsstrategien siehe Reinhard Bork, Die ,große Vorlesung“ für Juristen - Handreichungen für eine Massenveranstaltung, JuS 1999, 413 (413 ff.).

58 Flender, Didaktik der Hochschullehre (o. Fn. 43), S. 170 (178); siehe zur Rolle der Dozentinnen und Dozenten auch Reich, Konstruktivistische Didaktik (o. Fn. 30), S. 22 ff.

59 Fritjof Haft, Einführung in das juristische Lernen, 6. Auflage, Bielefeld 1997, S. 62 ff. und $113 \mathrm{ff}$.

60 Vgl. Fritjof Haft, Juristische Lernschule, München 2010, S. 98 f. 


\section{Auf dem Weg zur rechtswissenschaftlichen Didaktik}

Dies schlägt den Bogen zur rechtswissenschaftlichen (Fach-)Didaktik: Zum gegenwärtigen Zeitpunkt ist unklar, auf welche Weise sich der beste Lernerfolg bei den Studierenden erzielen lässt. Es lässt sich eben nicht sagen, welche Determinanten des didaktischen Handlungsspielraums die Entscheidung für die „Normalfallmethode“ begründen. Die Lehrperson muss sich deshalb im Augenblick bei der Wahl der richtigen Vermittlungsmethode noch auf ihre Intuition verlassen. Denn wissenschaftliche Erkenntnisse über den Lehr-/Lernprozess im juristischen Studium und damit über die ErschlieBung des didaktischen Handlungsspielraums in einer Vorlesung liegen noch nicht vor. Damit wird die Notwendigkeit einer wissenschaftlichen Perspektive auf die Rechtslehre zur Evidenz gebracht. Erst eine solche ermöglicht die Beurteilung, ob eine „Misere in der Rechtslehre" zu beklagen ist. 